\title{
LA MEDICALIZACIÓN DEL SUICIDIO EN LA ESPAÑA DEL SIGLO XIX: ASPECTOS TEÓRICOS, PROFESIONALES Y CULTURALES
}

\author{
José Javier Plumed Domingo \\ Hospital La Fe, Valencia \\ Luis Rojo Moreno
}

Universidad de Valencia

\section{RESUMEN}

El presente artículo analiza el proceso de medicalización del suicidio en España en el siglo XIX. Describe el paso de la concepción del suicidio como acto libre del sujeto a una conducta patológica susceptible de tratamiento por parte de la Medicina Mental. Frente a este nuevo modelo, otras posiciones conservadoras provenientes de la Medicina y el Derecho siguieron defendiendo el esquema tradicional.

El interés inicial de los médicos mentalistas por los aspectos sociales del suicidio se fue desarrollando durante este período. El hecho social que se invocó con más frecuencia fue la pérdida de ideas religiosas, lo que era comprensible teniendo en consideración la gran importancia que la religión tuvo en la ciencia y sociedad españolas. Con la introducción de la teoría de la degeneración esta conducta acabó formando parte del cuerpo de «enfermedades sociales» de la España de fin de siglo.

PALABRAS CLAVE: Suicidio. Siglo XIX. España. Nosología. Etiología.

\section{THE MEDICALIZATION OF SUICIDE IN $19^{\mathrm{TH}}$ CENTURY SPAIN: THEO- RETICAL, PROFESSIONAL AND CULTURAL ASPECTS}

\section{ABSTRACT}

This paper analyzes the medicalization process of suicide in Spain during the $19^{\text {th }}$ century. It describes the transition of suicide seen as an act of free will to a model, developed by mental doctors, which considered it a pathological behavior. Against this model, other conservative positions from the fields of Law and Medicine continued to defend the traditional view.

The initial interest of mental medicine regarding the social aspects of suicide was developed during this period. The social factor that authors considered to be the most influential to suicide was the loss of religious ideas, which was understandable considering that religion was very pre- 
sent in Spanish science and society throughout the century. During the last decades of the $19^{\text {th }}$ century, with the introduction of the degeneration theory in Spain, this behavior was finally included among the «Social Diseases» group

KEY WORDS: : Suicide. Nineteenth century. Spain. Nosologý. Etiology.

\section{INTRODUCCIÓN}

La historiografía del suicidio explica cómo en el siglo XIX se consolidó un proceso de transición en su concepción, desde la visión teocrática que criminalizaba las conductas autodestructivas hasta un modelo científico-médico de esta conducta.

Para justificar este proceso de transición, se ha apelado a factores de tipo profesional, social y teórico.

El posicionamiento de distintos colectivos profesionales respecto al suicidio tuvo gran influencia en su proceso de medicalización. En Inglaterra, M. McDonald estudia cómo los tribunales de justicia participaron en el cambio de concepción en la conducta estudiada durante el siglo XVIII. El embargo de los bienes del difunto, la consecuencia legal que en aquel momento histórico suponía el suicidio, sufría una oposición social cada vez más intensa. Ello llevó a un espectacular incremento de los fallos judiciales en que se consideraba al difunto víctima de una enfermedad mental y libre de pena, lo que contribuyó a la transición del concepto del suicidio como un acto libre a una enfermedad mental ${ }^{1}$. Para el caso de Francia, J. Goldstein pone como causa principal del cambio conceptual del suicidio a la consolidación de la especialidad psiquiátrica. Describe la autora cómo la nueva especialidad buscó la forma de legitimarse socialmente a través de distintos medios: la influencia como peritos en los juicios criminales, la capacidad de estructurar «científicamente» los fenómenos mentales mediante clasificaciones, sustituir el cuidado espiritual antes concedido por la iglesia por una atención médica populista y, en el caso del suicidio, cambiar uno de los pecados castigados socialmente por una enfermedad mental sin implicaciones morales ${ }^{2}$.

Un lugar común en la literatura médica de la época era relacionar los cambios sociales y políticos con el aumento de los casos de suicidio. Así, la transición del Antiguo Régimen a la nueva sociedad burguesa y liberal se consi-

1 MC. Donald, M. (1989), The medicalization of suicide in England: laymen, physicians and cultural change, 1500-1870. The Milkbank Quaterly, 67, Suppl. 1, pp. 69-91.

2 Goldstein, J. (2001), Console and classify. Chicago, Chicago Press, p. 383. 
deró la causante de nuevos males, en los que el suicidio ocupó un lugar preeminente. Entre los cambios más llamativos relacionados con la conducta autodestructiva estuvo el proceso de secularización de la sociedad, que supuso un enfrentamiento de la burguesía liberal con las fuerzas políticas conservadoras y con la Iglesia. Para el caso de Francia, J. McManners señala la gran importancia que tuvo la crítica constante por parte del poder eclesiástico a la ola de seglarismo que se estaba viviendo en Europa durante el siglo XVIII y que recurría al supuesto incremento de las tasas de suicidio como una de las señales más evidentes de este proceso. Ante las críticas, las fuerzas liberales empezaron a sostener la noción de que el suicidio se trataba de una enfermedad mental para defenderse ante las críticas constantes que sugerían que el suicidio era una consecuencia social más de la ideología ilustrada ${ }^{3}$.

Una de las visiones más difundidas sobre la razón que llevó al desarrollo y consolidación del proceso de medicalización del suicidio está relacionada con el papel conferido a la psiquiatría por las nuevas fuerzas sociales para actuar coercitivamente sobre los grupos que supusieran un peligro al orden burgués. En este sentido, Z. Cahn sugiere que el cambio conceptual del suicidio estuvo ligado a la aparición del movimiento romántico. Dado que la característica más señalada de este movimiento fue la apología del individualismo y la libertad, de la que el suicidio fue la muestra más característica, recibió por parte de la clase médica y otros grupos sociales una respuesta conservadora, en la que se consideraba esta visión como subversiva y, secundariamente, enfermiza ${ }^{4}$. Lieberman comparte de esta visión. De acuerdo con el modelo propuesto por Foucault, la psiquiatría participó en el proceso de control social en la transición del Antiguo Régimen a la sociedad moderna, en la medida en que convergieron sus intereses profesionales y los de la nueva clase dominante, la burguesía. Los frenópatas extendieron la idea de la existencia de una epidemia suicida causada por la conmoción debida a los cambios políticos y sociales, que eran distintos a los valores tradicionales de la Iglesia Católica5.

En el siglo XIX hubo un intenso debate psiquiátrico sobre la naturaleza del suicidio apoyado en un nuevo modelo de individuo. Las nuevas corrientes románticas, espiritualistas e idealistas concibieron un nuevo modelo de sujeto

3 MCManners, J. (1981), Death and the Enlightenment: changing attitudes to death among Christians and unbelievers in Eighteenth century France. Oxford, Oxford University Press, pp. 409-437.

4 CAHN, Z. (1998), Suicide in French thought from Montesquieu to Cioran. New York, Peter Lang Publising, pp. 123-174.

5 Lieberman, L. (2003), Leaving you. The cultural meaning of suicide. Chicago, Ivan Dee, pp. 22-24. 
que propiciaba la introspección y la reflexividad. Esto implicó un cambio en la concepción de la conducta humana y llevó a establecer cambios en los modelos y tratamientos psiquiátricos ${ }^{6}$. Hubo un intento de aproximación naturalista y científico al comportamiento humano, que apartó definitivamente al hombre de los modelos teológicos y metafísicos y lo consideró susceptible de estudio científico mediante la observación. Este nuevo yo reflexivo fue conceptualizado, de acuerdo con el esquema tomado de la Ilustración, en base a su oposición a la influencia patógena de las pasiones exacerbadas, que ponían en peligro permanente al individuo que surgió de esta sociedad en transición ${ }^{7}$. En la segunda parte del siglo XIX hubo un proceso de modificación de este esquema de persona y se fue definiendo un modelo somaticista y mecánico de sujeto $^{8}$, lo que influyó notablemente en la consideración del suicidio por parte de la medicina mental de la época.

La medicalización del suicidio exigía un desarrollo teórico y una nosología que permitiese definirlo como enfermedad mental, de la misma forma que sucedía con otras conductas socialmente inaceptables ${ }^{9}$. Este cuerpo teórico se desarrolló fundamentalmente en Francia, aunque hubo aportaciones desde otros países como Inglaterra, en que autores como Prichard y Maudsley tuvieron un importante papel en la definición médica de los trastornos de la voluntad ${ }^{10}$.

En una reciente revisión sobre el tema, R. Healy considera que para entender históricamente el suicidio se requiere su estudio en cada uno de los países europeos y llama la atención sobre la ausencia de estudios sobre los países de Europa del Sur, entre los que se encuentra España ${ }^{11}$. En su estudio sobre la historiografía española, Lázaro y Bujosa señalan la ausencia de estudios con-

6 HuERTAS, R. (2010), Locura y subjetividad en el nacimiento del alienismo. Releyendo a Gladys Swain. Frenia, 10, pp. 11-27.

7 WeIner, D. (1990), Mind and body in the clinic: Philippe Pinel, Alexander Crichton, Dominique Esquirol and the birth of psychiatry. En: RouSSEAU, G (Ed), The languages of the psyche. Mind and body in Enlightenment thought. Berkeley: California Press, pp. 331-401.

8 Álvarez, R; Huertas, R; Peset, J. (1993), Enfermedad mental y sociedad en la Europa de la segunda mitad del siglo XIX. Asclepio, 45 (2), 41-60.

9 MARTínez PÉREZ, J. (1996) Catalogando la diversidad del comportamiento humano: la nosología francesa decimonónica ante las conductas delictivas (1800-1855), Asclepio, 48 (2), pp. 87-114.

10 GATES, B.T. (1988) Victorian suicide. Mad crimes and sad stories. New Jersey, Princeton University Press.pp. 12-22.

11 HEALY R (2006) Historiographical reviews: suicide in early modern and modern Europe. The Historical Journal, 49, 3, pp. 903-19. 
ceptuales sobre el tema ${ }^{12}$. Este trabajo es una aportación en este hueco historiográfico y trata de analizar el proceso de la medicalización del suicidio en la España del siglo XIX.

\section{EL SUICIDIO COMO PROBLEMA DE CONDUCTA NO PSIQUIÁTRICO}

Uno de los debates más importantes sobre el suicidio en la literatura psiquiátrica del siglo XIX fue si debía ser considerado como una enfermedad mental o como el acto voluntario de un individuo mentalmente sano. Para G. Berrios y M. Mohanna, sobre 1880 triunfó en Francia la «Standard view», según la cual el suicidio a veces es consecuencia de la enfermedad mental y a veces no lo es $^{13}$. Estos autores cuestionan la concepción tradicional que vinculaba el pensamiento de Esquirol a un modelo organicista que consideraba la conducta suicida en último término como consecuencia de una patología cerebral. Si bien entendemos como ciertas dichas consideraciones, lo cierto es que el modelo propuesto por Esquirol para conceptualizar el suicidio se aproxima antes al organicismo que a una visión en la que el libre albedrío del sujeto sea acepta$\mathrm{da}^{14}$. Un modelo alternativo vino propuesto por la postura de Brierre de Boismont, cuyo libro «Du suicide et de la folie suicide» tuvo un gran predicamento en España, fundamentalmente entre los psiquiatras de orientación espiritualista que desarrollaron su trabajo en el segundo tercio del siglo XIX. Aunque Pedro Mata consideraba que fue el autor que logró el triunfo de la «Standard View» ${ }^{15}$, su obra se ligó a una crítica de la visión del suicidio como enfermedad mental y fue una de las autoridades más frecuentemente citadas en referencia al tema. Aunque su libro no fue traducido, hubo comentarios en la

12 LÁzAro, J; Bujosa, F (2000) Historiografía de la psiquiatría española. Madrid, Triacastela, p. 172.

13 Berrios, G.E.; Mohanna, M. (1990), Durkheim and French psychiatric views on suicide during the 19th century. A conceptual history. British Journal of Psychiatry, 156, pp. 1-9.

14 Esquirol escribe en su Tratado: «Puesto que el suicidio es casi siempre una enfermedad no debe ser castigado, la ley no impone penas sino a los que comenten actos en plena razón. Creo haber demostrado que el hombre no atenta contra sus días sino cuando está delirante y que los suicidas son enagenados». ESQUIROL, J.E. (1857), Tratado completo de las enagenaciones mentales consideradas bajo su aspecto médico, higiénico y médico-legal,. Madrid, Imp. De Gómez Fuentenebro, Tomo I, p. 285.

15 MatA, P. (1875) Tratado de Medicina y Cirugía legal. Teoría y práctica. Madrid, Bailly-Bailliere, p. 482. 
literatura médica referenciando las reediciones francesas ${ }^{16}$ y se tradujo un trabajo del autor acerca de esta materia ${ }^{17}$. Brierre de Boismont creía que sostener que el suicidio era siempre un síntoma de locura implicaba negar la importancia en la conducta de las ideas y de las creencias. Interpretaba la tendencia de algunos frenópatas a considerar que el suicidio era siempre consecuencia de la locura como un sesgo profesional, ya que su práctica les obligaba a valorar enfermos mentales y no personas sanas, y alertaba contra la tendencia a considerar al ser humano como un grupo uniforme, sin importantes diferencias individuales ${ }^{18}$. En su análisis sobre el «fastidio de vivir», consideraba que se trata de un problema emocional complejo, relacionado tanto con los cambios sociales (cambios políticos, secularización) como con el nuevo modelo de sujeto, que ejemplificaba el Werther de Goethe. Para Brierre de Boismont este personaje se caracteriza por: su imaginación desenfrenada ${ }^{19}$, sus deseos hacia un objeto desconocido y por su actitud más centrada en la reflexión que en la acción ${ }^{20}$. Por otro lado, la hipertrofia de un yo más volcado a la reflexión, al pensamiento y a la búsqueda de la satisfacción que a actuar un ideario social establecido se ligaba al movimiento romántico, que se consideró ideológicamente peligroso. Estos factores, para Brierre de Boismont, no radicaban en una alteración del juicio de base somática, sino en una desviación del carácter condicionada por el libre albedrío del sujeto, que se decide por tomar una dirección vital equivocada. De hecho, aunque aceptaba los casos de locura entre los suicidas, concluye su trabajo afirmando que «el disgusto de la vida es frecuentemente una causa de suicidio sin que haya síntomas de enajenación mental» ${ }^{21}$.

16 Nieto y Serrano, M. (1865) El suicidio y la locura suicida. Siglo Médico, 12, p. 472.

17 Brierre De Boismont, A. (1854), Del fastidio de la vida, Boletín de Instituto Médico Valenciano, 5: pp. 158-61, 171-7, 189-93, 201-4, 217-22, 233-36.

18 Brierre De Boismont, A.(1856) Du suicide et de la Folie suicide. Paris, Baillière, pp. 482-6.

19 La imaginación fue considerada por el pensamiento ilustrado como una de las facultades más importantes, con la razón y la memoria. Se consideró que era, a su vez, la más peligrosa, en cuanto su carácter subjetivo le hacía ser fuente de errores y enfermedades, tanto a nivel psíquico como físico. En la medicina mental española del siglo XIX se recogió esa tendencia, de modo que los médicos que se planteaban los factores ligados a la causalidad de la enfermedad mental consideraban este factor como importante y, desde el higienismo, se planteaban medidas sociales para limitar el alcance de esta facultad. Ver: NovELLA, E. (2010), La higiene del yo: Ciencia médica y subjetividad burguesa en la España del siglo XIX. Frenia, 10, 49-74, pp. 55-61.

20 BRIERRE DE BOISMONT, A. (1854), pp. 175-6.

21 BRIERRE DE BOISMONT (1854), p. 236. 
En la medicina mental española del segundo tercio del siglo XIX pervivían activos los autores espiritualistas, que vieron con recelo los primeros intentos de incorporación de la medicina mental europea, fundamentalmente en lo que coresponde a los modelos cercanos al materialismo. De la misma forma que en Francia, pero con mucha mayor virulencia, hubo una agria polémica entre estos y los autores materialistas ${ }^{22}$. Independientemente de que ambos modelos teóricos aceptaban la existencia de un sustrato orgánico en la locura, sus planteamientos ideológicos estaban claramente diferenciados, en la medida en que los espiritualistas consideraban que la realidad psíquica era independiente de las propiedades materiales del cuerpo y no podía comprenderse su funcionamiento simplemente mediante el estudio de la fisiología. El materialismo se relacionó con el progresismo y el liberalismo y se reivindicó como una amenaza al estado social durante todo el siglo ${ }^{23}$.

Los autores espiritualistas aceptaban un modelo dualista del psiquismo humano, lo que permitía compatibilizar el modelo de sujeto con la idea de libre albedrío propuesta por el dogma católico. Hemos de recordar que el peso de la Iglesia Católica en la vida social y política española fue enorme y tuvo un gran peso en el desarrollo de diversas áreas de la ciencia en nuestro país durante todo el siglo. Fueron conocidos los virulentos debates en foros académicos y públicos entre los defensores del catolicismo conservador y los introductores de las nuevas ideas científicas que fueron consideradas peligrosas ideológicamente desde la Iglesia. Los primeros adoptaron una posición cada vez más defensiva y hostil hacia los planteamientos filosóficos materialistas y hacia la introducción de modelos científicos que pudieran cuestionar el dogma católico en distintas ramas de la ciencia, como las nuevas doctrinas evolucionistas ${ }^{24}$.

Para los psiquiatras de orientación espiritualista en España, la defensa del libre albedrío fue un punto ideológico fundamental. En el caso del suicidio, un acto calificado por la Iglesia como pecado mortal, la defensa de un modelo dualista que no considerase un determinismo orgánico en la conducta del paciente era esencial. Este modelo estaba condicionado por el esquema voluntarista de Maine de Biran, según el cual la salud mental dependería de un equilibrio de fuerzas entre la voluntad libre del ser humano, conocida como «interioridad», frente a las fuerzas orgánicas, ligadas al elemento pasional.

22 Goldstein (2001), pp. 240-75.

23 Sobre la relación entre ciencia positivista y liberalismo ver: NuÑEZ, D (1975), La mentalidad positivista en España: desarrollo y crisis. Madrid, Tucar.

24 Pelayo, F. (1999), Ciencia y creencia en España en el siglo XIX. Madrid, CSIC. 
Aunque ambas fuerzas interaccionaban no lo hacían recíprocamente y tanto el yo voluntario como el libre albedrío no estaban plenamente condicionados por lo físico ${ }^{25}$. Para Maine de Biran, la locura se definía por la pérdida de la libertad y de la conciencia del yo, fenómenos que no ocurrían sistemáticamente en el caso del suicida, siguiendo el esquema ya visto en Brierre de Boismont. De acuerdo con este planteamiento, Joaquín Quintana publicó una ponencia en el Primer Congreso Español de Medicina, donde exponía un punto de vista psicologicista de la enfermedad mental. Negaba que las pasiones fuesen una función meramente orgánica y definía la monomanía de forma coherente con el modelo espiritualista: «fenómeno psicológico, acompañado de representaciones pasionales anormales, que determinan anormalmente las afirmaciones de conciencia en una dirección especial» ${ }^{26}$. F. Castellví y Pallarés hacía suyo este pensamiento reconociendo en el hombre un «principio de unidad inmaterial, espiritual, dotado de facultades propias que no son del organismo» y caracterizado por la conciencia del yo, que recibe impresiones del cerebro aunque depende de sí mismo para pensar ${ }^{27}$. En su Memoria sobre el Suicidio, si bien al hablar de los suicidas que ejecutan el acto de forma aparentemente libre afirmaba la probable existencia en cada caso de «una pasión que, sorda y lentamente, haya sobreexcitado su cerebro produciendo una monomanía», acababa por desviarse del criterio unitario y aceptaba un modelo que relacionaba con Descuret: «casi todos los que atentan contra sí hay monomanía suicida; pero que no deja de haber personas que se maten en la plenitud de la razón» ${ }^{28}$.

J.B. Descuret, fue un autor de gran influencia en España. Médico y doctor en Letras, mostraba un modelo de individuo bien definido, en el que las pasiones, enfermizas por naturaleza, han de ser dominadas en todo momento por una razón sólida capaz de someter las fuerzas de nuestra naturaleza interna. En su libro, La medicina de las pasiones $^{29}$, entre las múltiples consecuencias enfermizas del descarrío pasional señalaba al suicidio, al que definía como

25 Goldstein (2001), pp. 260-1.

26 QuintanA, J. (1863), Discurso pronunciado sobre la «pasión y la locura» en la Real Academia de Medicina de Madrid. Siglo Médico, 10, 341-4; 357-9; 373-5; 390-2, p. 390.

27 Castellví y Pallarés (1845), Memoria sobre el suicidio. Boletín del Instituto Médico Valenciano, 2, 466-8; 473-5; 482-5; 493-9; 505-9; 513-20 ,p. 483.

28 CASTELlVí y PALlarÉs, F (1845), p. 496.

29 Se tradujo en dos ocasiones y tuvo numerosas reediciones; la edición más conocida fue traducida por Pedro Felipe Monlau en 1842; la otra por Francisco de Borja García Blanco. Sobre esta obra se ha publicado: DIÉGUEZ, A.(2010) Perspectiva sobre las pasiones en la España del período Romántico. Frenia, 10, pp. 29-48. 
«triple atentado contra la moral, contra Dios y contra sí mismo» ${ }^{30}$. Consideraba el acto como consecuencia de un «delirio de las pasiones» ${ }^{31}$ y hablaba de estado mórbido, aunque consideraba que el suicida conservaba su capacidad de decisión. Este modelo de Descuret, aplicado a la conducta suicida, tuvo un notable éxito entre autores de orientación espiritualista. V. Moreno y López citaba a Descuret, señalando que las pasiones no estaban relacionadas con el alma ni con el cuerpo, sino que serían «independientes de la materia a que resisten», aceptando un dualismo psíquico ${ }^{32}$. Los autores espiritualistas fueron perdiendo predicamento en la medicina mental española a partir de la segunda mitad del siglo XIX. Sin embargo, en determinados colectivos siguió manteniéndose el modelo de conducta suicida como un acto libre del sujeto no ligado a la enfermedad mental.

Desde el Derecho, hubo una actitud inicialmente reacia a aceptar la medicalización de conductas socialmente inaceptables que, hasta el siglo XIX, habían estado determinadas por la competencia de jueces y abogados. Sin embargo, en Francia hubo a partir de la tercera década del siglo una significativa aproximación entre jueces y frenópatas, en la medida en que se fueron aceptando en los tribunales diagnósticos de monomanía ${ }^{33}$. Pero hubo importantes reticencias como la defendida por Elias Regnault, un autor frecuentemente citado en España, que fue uno de los legistas opuestos al diagnóstico de monomanía. En un libro sobre esta enfermedad consideraba el suicidio como la más alta expresión de la libertad humana. Distinguía al hombre del animal en cuanto podía recurrir al suicidio: «el animal pasivo no puede responder a las torturas de la enfermedad más que una planta» ${ }^{34}$. Esta visión de individuo libre fue aceptada por un gran número de legistas que escribieron sobre el suicidio en España.

30 DESCURET, JBF. (1857) La medicina de las pasiones o las pasiones consideradas con respecto a las enfermedades, a las leyes y a la religión. Barcelona, Imprenta Pablo Riera (2 ${ }^{\mathrm{a}}$ edición) original de 1841, p. 364.

31 Aquí hemos de recordar lo ambiguo del término «délire» en la psiquiatría francesa, que no se puede identificar con la definición del término delirio en los términos en que lo conocemos ahora, sino que hay que entenderlo como un acto pasional que no está estrictamente fuera del control voluntario, aunque lo debilita. Ver: BERRIOS, G.E.; MOHANNA, M (1996) Suicidal behaviour. In: Berrios, GE; PORTER, R. A history of clinical psychiatry. London, Athlone Press, 612-24, p. 615.

32 MORENO y LÓPEZ, V. (1864) ¿Cuáles son los caracteres diferenciales de la monomanía y la pasión?. Toledo, Imp. López Fando, p. 26.

33 Huertas, R (2005),El siglo de la clínica. Madrid, Frenia, p. 77.

34 Regnault, E. (1830), Nouvelles réflexions sur la monomanie homicida le suicide et la liberté morale. Paris,: Baillière, p. 99. 
La legislación española que seguía vigente durante el XIX fue la ley 15, título XXI, libro 12 de la Novísima Recopilación, que ordenaba que los bienes del suicida fueran incautados. Sin embargo, no se aplicaba sistemáticamente porque estaba aceptado por los Tribunales lo injusto de esta ley ${ }^{35}$. Aunque el papel en las sentencias fue nulo, la reticencia por parte de abogados y de determinados médicos legales a aceptar el diagnóstico de monomanía suicida fue significativa y estaba relacionada con la adscripción a los modelos conservadores que consideraban algo escandaloso aceptar que el suicidio podía ser un acto sin connotaciones morales peyorativas.

Aquellos que consideraban al suicidio un acto de locura defendían mantener fuera cualquier medida legal coercitiva. Sin embargo, desde el campo del Derecho y de la Medicina Legal más conservadora se propusieron cambios legislativos para frenar una conducta considerada como un peligro social desde el punto de vista moral. R. Ferrer y Garcés, catedrático de Medicina Legal de la Universidad de Barcelona, defendía la imposibilidad de considerar que todo acto suicida era consecuencia de un estado de enfermedad y lo consideraba un subterfugio para impedir la estigmatización de aquel que había cometido este acto. Además, proponía retomar medidas punitivas para frenar estos comportamientos: «podría afectar la buena memoria del suicida, cubriéndola de una mancha de infamia; y esto tal vez sería suficiente para contener el brazo que había armado la desesperación u otra causa igualmente lamentable» ${ }^{36}$. F. Álvarez Arenas, juez de Primera Instancia, exponía en su libro la actitud de varios jueces respecto al tema. Si otro jurisconsulto, Salas, rechazaba la condena del acto suicida porque de él no se derivaba un mal para el individuo y el juez Pacheco lo consideraba consecuencia de un acto de enajenación, este autor mostraba su queja de que «ya tenemos al jurisconsulto español participando enteramente de las ideas de los filósofos que atribuyen el suicidio a la demencia» ${ }^{37}$ y pensaba que se trataba, simplemente, de una invención para defender el suicidio. También proponía retomar la práctica de la infamia pública sobre el cuerpo del suicida y así volver a aplicar la ley 15, ya citada. Incluso ya en el principio del siglo XX un jurista, Sicars y Salvadó considera-

35 Sicars y Salvadó, N. (1902), El suicidio jurídicamente considerado. Barcelona, Librería Barcelonesa, p. 141.

36 FERRER Y GARCÉS, R. (1847), Tratado de Medicina Legal o exposición razonada de las cuestiones jurídico-médicas que se exponen en los tribunales de justicia. Barcelona, Imp. Pablo Riera, pp. 338-342.

37 Álvarez Arenas, F. (1859), Cuestiones filosófico-politico-legales sobre los delitos del suicidio y del duelo. Madrid, Imp. De la Revista de la Legislación, p. 58. 
ba que el Código Penal de 1870 no hubiese reformado la legislación frente al suicidio suponía que «los legisladores españoles se han visto envueltos en las ideas que dominan en nuestros días y no parece sino que nos avergonzamos de seguir las tendencias de determinados filósofos»» ${ }^{38}$. Y aunque reconocía la postura de destacados juristas en contra, proponía volver a las medidas judiciales del Antiguo Régimen ya citadas, incluso el castigo del cadáver del suicida.

De la misma forma, los médicos de orientación católica y conservadora, aunque no dedicados a la medicina mental, se opusieron a la medicalización del suicidio y mantuvieron la idea católica sobre la libertad individual del sujeto que comete este acto. En su tesina doctoral, E. Gaspar hablaba de la importancia de combatir al suicidio y del hecho que «nuestra Santa madre la Iglesia Católica anatemiza justamente el suicidio como acto de rebelión contra la voluntad divina» ${ }^{39}$. En una revista de orientación católica, aunque empezaba aceptando que el suicidio podía ser una manifestación de conducta consecuencia de una disfunción cerebral, Carbonell y Solés defendía la libertad moral basada en los principios católicos y creía que el acto suicida es consecuencia de una falta de principios morales de base religiosa, que degeneran en un estado enfermizo aunque prima la libertad del sujeto en el acto. ${ }^{40}$

\section{EL SUICIDIO COMO ENFERMEDAD MENTAL}

Desde las primeras décadas del siglo XIX hubo autores que definieron al suicidio desde el punto de vista médico. La tendencia por parte de autores de orientación liberal a medicalizar las conductas que tradicionalmente habían sido definidas desde el prisma filosófico, moral o legal fue progresiva pero constante, y resultaba enormemente tentador hacerlo en un área tan controvertida. En un artículo anónimo el autor consideraba que «son muy pocas las excepciones de la regla que considera al suicidio como el resultado de una demencia o locura»; "o como el efecto de aquellas pasiones vivas, violentas y casi irresistibles que no pueden menos de ocasionar un trastorno intelectual» ${ }^{41}$. En su obra Elementos de Medicina y Cirugía, P. Peiró y J. Rodrigo,

\footnotetext{
38 SiCARS Y SALVADÓ (1902), p. 143.

39 Gaspar, E. (1896), El suicidio. Granada, Imp. El Pueblo, p. 3.

40 Carbonell y Soles, F (1898), Reflexiones sobre el suicidio. El Criterio Católico de las Ciencias Médicas, I, 8, pp. 260-7.

41 ANONIMO (1821), Consideraciones médico legales sobre la desesperación y el suicidio. Décadas Médico-Quirúrgicas, 2, 49-63, p. 49.
} 
en relación al suicidio, se felicitaban de la falta de medidas legales frente al suicida, dado que era un trastorno mental: «el suicidio no tiene, pues, ningún objeto real; no tiene más otro que es ilusorio» «Hemos probado que era el resultado de una enfermedad. Esta acción carece pues de libertad y no puede ser efecto de la voluntad de un hombre sensato ${ }^{42}$. R. Nadal y Lacaba también consideraba que el suicidio era un signo inequívoco de enfermedad mental. Para este autor, «en los diferentes modos de quitarse el hombre su preciosa existencia no hay ni fuerza, ni debilidad, ni valor ni cobardía; solamente sí domina en la constitución física una afección crónica o aguda, que no deja de distinguirse por los fenómenos morales o físicos que anteceden» ${ }^{43}$. Ilustrativo de este paradigma fue una reunión que tuvo lugar en el Instituto Médico Valenciano, donde se concluyó que «el suicidio, entre los que profesan la religión cristiana, es necesariamente una monomanía» ${ }^{44}$.

Uno de los modelos materialistas más representativos en la primera mitad del siglo XIX fue el movimiento frenológico. Esta corriente teórica consideraba que el cerebro es un conjunto de órganos que contribuyen a la función global del psiquismo y la irritación de cada una de sus partes daría lugar a una manifestación conductual diferente. En el caso del suicidio, la frenología definió un modelo organicista y mecánico de esta conducta. Pers y Ramona pensaba que la causa del suicidio se debía a un trastorno debido a un escaso desarrollo del órgano cerebral de la conservatividad y muy pronunciado del órgano de la destructividad ${ }^{45}$. De la misma opinión era Cubí y Soler, que defendía que este rasgo se unía a las alteraciones en la circunspección, la tendencia a la autoprotección, que aparece en el área posterolateral de la cabeza $\mathrm{y}$, si estaba deformada, aumentaba la tendencia al suicidio ${ }^{46}$.

Pedro Mata, catedrático de Medicina Legal, fue uno de los médicos más importantes del período. Dentro de su amplio campo de actividades prestó un notable interés a la medicina mental. Estuvo muy influido por la doctrina frenológica y reconoció su inspiración en la obra de Esquirol, del que tomó

42 PeIRÓ, P.; Rodrigo, J. (1844), Elementos de Medicina y Cirugía Legal, arreglados a la legislación española. Zaragoza,Imp. Mariano Peiró, p. 108.

43 NadAl y LaCABA, R (1844), Suicidios. Barcelona, Imp. Antonio Brusi, p. 5.

44 AVEllan, R (1845), El suicidio, ¿es compatible con la integridad de las funciones intelectuales? Boletín del Instituto Médico Valenciano, 2, 9-10, p. 9.

45 Pers y RAmona, M. (1849), Manual de frenológía al alcance de todos. Barcelona, José Tauló, p. 333.

46 CUBí Y SOLER, M. (1849), Elementos de frenología, fisonomía y magnetismo humano,en completa harmonía con la espiritualidad, libertad e inmortalidad del alma. Barcelona, Imp. Hispana, pp. 85-6. 
tanto su esquema nosológico como su orientación organicista. Así, fue uno de los introductores del concepto de monomanía en España, que defendía para explicar conductas anómalas como «locuras de amor, ambición, de fanatismo religioso, político, de persecución, de homicidio, suicidio, etc» ${ }^{47}$. Fue constantemente acusado de materialismo y ateísmo por círculos católicos radicales y respondió a las críticas con un libro, donde hacía referencia al problema del suicidio ${ }^{48}$. Describía al sujeto que sufría este trastorno como víctima de pasiones enfermizas, en lo que denominaba «delirio del instinto», ajustándose al concepto de monomanía instintiva de Esquirol ${ }^{49}$ : «hay sujetos que luchan largos años contra su tendencia al suicidio»; «mas son manzanas que aunque bellas al exterior llevan dentro un gusano que las devora ${ }^{50}$, en una referencia inequívoca al carácter endógeno e involuntario de su origen. Incluía al suicidio entre las distintas modalidades de trastornos de comportamiento que merecían la categoría de locura monomaníaca y hacía una alusión al famoso caso de Juana Sagrera ${ }^{51}$ como queja de la falta de reconocimiento social de este nuevo diagnóstico de locura que pugnaba por validarse en los tribunales ${ }^{52}$. En su Tratado de Medicina Legal mantenía una postura ambivalente con respecto a la consideración del suicidio como enfermedad mental. Si bien defendía inicialmente la «Standard view», por otro lado comentaba que «el instinto de la propia conservación es muy poderoso en el hombre; y obrar contra su ten-

47 Mata, P. (1858), Tratado de la razón humana con aplicación a la práctica del foro. Madrid, Bailly-Bailliere, p. 483.

48 Mata, P. (1868), De la libertad moral o libre albedrio. Madrid, Bailly-Bailliere, p. 9.

49 Recordemos que el modelo propuesto por Esquirol implicaba siempre la existencia de delirio, aunque afectaba únicamente a la voluntad, siendo detectable por el comportamiento del sujeto. Ver: HUERTAS (2005), pp. 72-74.

50 MATA (1868), p. 130.

51 Sobre este famoso caso está publicado: CuñAT, M. (2007) Las cartas locas de doña Juana Sagrera. Frenia, VII, 89-107; REY, A; PlumeD, J (2004) La verdad sobre el «caso Sagrera». En: Álvarez J.M., Esteban, R. (coords.) Crimen y Locura. Madrid, AEN, pp. 85132. Recordamos que Pedro Mata fue uno de los peritos del caso, en el que intentó sostener sin éxito el diagnóstico de monomanía e incapacidad de la supuesta enferma.

52 Para llevar a cabo este proceso, la psiquiatría española empezó a contar tempranamente con el aparataje teórico procedente de Francia para diagnosticar y definir las conductas anómalas relacionadas con los trastornos de la voluntad, del que el concepto de monomanía fue el modelo más sobresaliente. Sobre el tema se ha publicado: MARTínEZ PÉREZ, J. (1996) Problemas científicos y culturales en la difusión de una doctrina psiquiátrica: la introducción del concepto de monomanía en España (1821-1864), En: ARQuIOLA, E.; MARTíNEZ PÉREZ, J. (Eds.), Ciencia en expansión. Estudios sobre la difusión de las ideas científicas en España (s. XVIII-XX), Madrid, Complutense, pp. 490-520. 
dencia tiene, en efecto, todo el sabor de una aberración, de una locura». Identificaba a los que supuestamente se suicidan en estado cuerdo como «víctimas de la violencia de la pasión que les domina y en esto se encuentra la lógica explicación de su atentado» ${ }^{53}$. Vemos que el modelo que describe tiende al somaticismo y a considerar al suicidio como la consecuencia irrefrenable de una irritación cerebral.

Desde el punto de vista profesional, la psiquiatría española vivió sus primeros intentos de institucionalización en la segunda parte del siglo. El primer paso en este proceso se ha considerado que corresponde a la escuela catalana, encabezada por Juan Giné y Partagás, la figura más importante de la psiquiatría española del siglo XIX ${ }^{54}$. Este autor, de ideología liberal y progresista, sostuvo una visión positivista de la enfermedad mental que casaba mal con el pensamiento conservador en varios aspectos fundamentales, siendo uno de los más importantes el libre albedrío, cuestión sobre la que la psiquiatría española vivió un intenso debate ${ }^{55}$. Los trastornos de la voluntad fueron en el siglo XIX uno de los aspectos psicopatológicos más importantes no sólo a nivel clínico $^{56}$ sino que, además, fueron claves en la intervención del psiquiatra en distintos campos de gran influencia social, como la Justicia y el Higienismo. Se ha descrito cómo uno de los primeros pasos en el proceso de institucionalización de la psiquiatría fue a través de los Tribunales de Justicia, de modo que la consideración del acto criminal como una conducta sólo evaluable por el psiquiatra se ha relacionado en España, como Goldstein lo hizo para Francia, con la necesidad de afirmación profesional del psiquiatra ${ }^{57}$. En el caso del suicidio, el debate estuvo ligado al interés de la psiquiatría de redefinir una conducta de gran impacto público y que fue progresivamente definida como enfermedad social a tratar, de acuerdo con los principios del Higienismo.

Aunque Giné y Partagás en su Tratado teórico-práctico de Frenopatología dedicaba un espacio a justificar la existencia del alma, probablemente para no ser cuestionado por su materialismo, describía en términos puramente físioló-

53 MATA (1875), p. 482.

54 Huertas, R. (2002), Organizar y persuadir. Madrid, Frenia, p. 77.

55 DiÉGUEZ, A. (2002), El problema del libre albedrío en el alienismo español. En: FUENTENebro, F; Huertas, R; VAliente, C.(Eds.), Historia de la psiquiatría en Europa, Madrid, Frenia,, pp. 137-46.

56 BERRIOS, GE; GILI, M. (1995), Will and its disorders: a conceptual history. History of Psychiatry, 6, 21, pp. 87-104.

57 CAmpos, R; MARtínez PÉrez, J; Huertas, R (2000), Los ilegales de la naturaleza. Medicina y degeneracionismo en la España de la Restauración (1876-1923), Madrid, Frenia, pp. 53-6. 
gicos el fenómeno voluntario, «la voluntad es expresión de un automatismo cerebral, cuyo origen, teniendo en cuenta los datos fisiológicos, debe referirse a las células de la zona cortical o de los cuerpos estriados» ${ }^{58}$, con lo que reducía el problema del libre albedrío a la expresión de un fenómeno neurobiológico. En las clases de psicopatología impartidas en su manicomio de Nueva Belén, Giné y Partagás sostenía la independencia de la voluntad frente a otras facultades y veía el suicidio como un acto de «heterabulia», definido como «actos impulsivos, ejecutados sin motivo intelectual previo y aun frecuentemente contra las aspiraciones, más o menos racionales, que nacen en la mente del enfermo» ${ }^{59}$. Su discípulo, Arturo Galcerán Granés, sostuvo un planteamiento radicalmente somaticista, recibiendo la influencia de la psicofisiología de Ribot y de su obra, «Las anomalías de la voluntad» ${ }^{60}$. Ya comentaba Galcerán en referencia al libre albedrío: «el libre albedrío es una ilusión» «es la volición un proceso puramente orgánico, mejor, un proceso cerebral, y como tal, un producto de correlación e integración de fuerzas materiales ${ }^{61}$. Para Galcerán Granés el suicidio, como el homicidio, sería una alteración de la voluntad, por exceso y consciente, en las que «la sensibilidad orgánica, transmitiendo potentes corrientes y el exagerado reflejismo cerebral, convirtiendo estas corrientes en voluntad fatal, es lo único enfermo» ${ }^{62}$.

Vemos cómo en la década de los 80 está consagrado desde la medicina mental un modelo del suicidio como enfermedad mental, causada por un sustrato orgánico alterado y que entraba directamente en la jurisdicción del especialista, a pesar de las críticas ya discutidas que venían desde otros campos.

\section{EL SUICIDIO COMO PROBLEMA SOCIAL}

Desde las primeras décadas del siglo XIX, los frenópatas consideraron que el suicidio tenía una notable raigambre social. Se especuló con que los cam-

58 Giné y PARTAGÁs, J. (1876), Tratado teórico-práctico de frenopatología. Madrid, Moya y Plaza, p. 183.

59 GinÉ y PARTAGÁs, J (1881), Aforística frenopática. Revista Frenopática Barcelonesa, 1, 41-5; 81-7; 121-4; 161-7; 201-6; 241-7; 281-7; 321-5; 361-5; 401-4; 444-5, pp. 361-63.

60 Ribot, Th. (1883), Les Maladies de la Volonté. Paris, Alcan.

61 GALCERÁN GRANÉS, A. (1884), El determinismo en la voluntad y el libre albedrío: crítica de ambas teorías. Revista Frenopática Barcelonesa. 3, p. 369.

62 GALCERÁn GRANÉs, A. (1884), Responsabilidad parcial de los enajenados. Datos prácticos para conocerla y graduarla. Gaceta Medica Catalana, 7, 580-90; 621-29; 648-57; 689$99 ; 714-22 ; 745-52$, p. 628 . 
bios sociales tenían una relación causal con el número de suicidios, lo que fue una señal de alarma sobre un supuesto estado enfermizo de la nueva sociedad. Ante este hecho la medicina mental tenía que dar una respuesta, con lo que la intención de los médicos del S XIX de tomar un papel cada vez más activo en la organización social se hacía presente en el caso del debate sobre el suicidio. No es casual que la personalidad más notable del higienismo en España, Pedro Felipe Monlau, se interesase por el suicidio en sus Tratados. En el caso de su libro «Elementos de Higiene Pública» planteaba que el suicidio era siempre un acto mentalmente enfermizo y, que en el caso de que los suicidas fallasen habría que tratarlos «como enfermos morales» y «quizás deberán ser pasados a un manicomio más bien que a una penitenciaría» ${ }^{63}$, con lo que incluía al suicida dentro de la esfera de acción del médico.

Ya Esquirol señalaba en su Tratado los factores sociales que estaban relacionados con el suicidio. Aquí distinguía entre aspectos individuales (edad, sexo, estado civil) y sociales. Entre estos, se destacaba tanto la influencia de la civilización como la pérdida de la religiosidad. La civilización como factor causal de la locura fue un tema recurrente. Claramente influido por el pensamiento de Rousseau, la idea de que la civilización producía una exaltación de las pasiones y un aumento del número de locos fue frecuentemente invocada. Además, constantemente se hacía referencia a citas estadísticas que justificaban con datos esta afirmación. De la misma forma, los cambios políticos (señalaba la Revolución) contribuían al proceso de excitación colectiva ya citado y aumentaban la frecuencia de monomanías, entre ellas el suicidio ${ }^{64}$, lo que dio lugar a un intenso debate en la psiquiatría francesa ${ }^{65}$. En España, muchos acusaban a los cambios producidos por la civilización, estimulante y excitante de las pasiones, de ser una causa frecuente de suicidio ${ }^{66}$. Así, Castellvi y Pallares lo relacionaba con el aumento de las necesidades consecuencia de la civilización moderna y la dificultad para satisfacerlas, consecuencia del egoísmo y de las condiciones sociales ${ }^{67}$. Nadal y Lacabra proponía medidas de higiene social para frenar los casos de suicidio, cuyo objetivo fuese desarrollar una clase media, de muy escaso peso en la España de la época, en que las

63 Monlau, P. (1847), Elementos de higiene pública. Barcelona, Imp. Pablo Riera, p. 760.

64 ESQUiROL, J.E. (1857), Tomo I, p. 155.

65 MARTínez PÉreZ, J. (2001), Suicidio, crisis política y medicina mental. Frenia, I (2), pp. 40-65.

66 MAestre De SANJuAn, A. (1851), ¿Qué causas conducen al hombre a poner fin a su vida?. Madrid, Imp. Del colegio de Sordomudos y Ciegos, p.15.

67 CASTElvi y Pallarés (1845), p. 506. 
tensiones económicas y sociales fuesen menos intensas: «Debe tender sin cesar a preservar la clase inferior de los vicios de la ignorancia y la miseria y a la clase opulenta de los de la insolencia y falso saber; procurando en aproximar las dos espresadas a la clase media en donde reina naturalmente el espíritu de orden, de trabajo, de justicia y de razón, porque en fuerza de su posición y de su interés directo está igualmente apartada de todos sus excesos» ${ }^{68}$.

La pérdida de valores religiosos fue una cita constante en los autores que alertaban sobre las causas sociales de suicidio. Brierre de Boismont opinaba que el mayor problema de la sociedad moderna estribaba en la pérdida del sentimiento religioso: «sociedades que envejecen, habiendo perdido las almas el sostén de la fe ${ }^{69}$. Si en países como Francia y Reino Unido el proceso de laicización de la sociedad estaba en pleno avance, en España fue conflictivo y difícil durante todo el siglo. Hubo una tendencia general en los autores a considerar los principios religiosos como la mejor preventiva para el suicidio. Maestre de San Juan planteaba, como prevención del suicidio, una educación basada en los más rígidos principios de religión y moral ${ }^{70}$. En su libro de Higiene Pública, Monlau consideraba que el mayor factor preventivo era una buena educación cristiana.: "desde el momento en que se enflaquecen las creencias religiosas» «»todo está perdido; no os extrañéis que el individuo se atreva a levantar una mano homicida contra sí mismo» ${ }^{71}$. De la misma forma, en su libro sobre la higiene individual Monlau referenciaba a Descuret, señalando que la pérdida de religiosidad era la mayor causa de suicidio ${ }^{72}$. Un autor autodenominado «Platón resucitado», en una revista médica de orientación católica, consideraba el suicidio como «delito social e individual y pecado»; «consecuencia de la poca solidez de su educación católica» ${ }^{73}$. Aunque vimos que los frenópatas sostenían una visión materialista y atea del problema del suicidio, para un gran número de autores provenientes del Derecho y otras ramas de la medicina la causa más importante del suicidio, mal epidémico de la España moderna, seguía siendo la pérdida de la fe católica ${ }^{74}$ y el argumento siguió teniendo un papel importante hasta final de siglo.

\footnotetext{
68 NADAl y LACABRA (1844), P. 13.

69 BRIERRE DE BOISMONT (1854), p. 190.

70 Maestre de SANJuAn (1851), p. 15.

71 MONLAU (1847), pp. 755-6.

72 Monlau, P (1864), Elementos de higiene privada o arte de conservar la salud del individuo Madrid, Imp. Moya y Plaza (cuarta edición), p. 392.

73 Platón Resucitado (1884), Una página de un libro antiguo. El Sentido Católico de las Ciencias Médicas, VI, 21, 317-323.

74 Álvarez Arenas (1859), p. 73; Arbunies (1882), p. 17.
} 
En el año 1843 el Ministerio de Gracia y Justicia publicaba datos epidemiológicos sobre los suicidios y tentativas, que empezó a hacerse de forma constante en la segunda parte del siglo. Los autores españoles recurrieron cada vez con más frecuencia a las cifras para justificar sus postulados y establecían una ligazón entre el crecimiento de las cifras del suicidio en diversos países europeos, fundamentalmente Francia, y la situación española, en la que las cifras aumentaban constantemente. Hubo una intensa discusión sobre las causas sociales que producían el aumento de suicidios. En este sentido, Ignagcio Valentí y Vivó consideraba que buen número de conductas aceptadas socialmente, entre las que señalaba: las lecturas eróticas, los espectáculos obscenos, la holganza, el mal ejemplo y la relajación de los vínculos familiares; tenían una implicación en los casos de suicidio, por lo que recomendaba su supresión o control ${ }^{75}$. Jimeno Agius señalaba que, entre los años 1859 y 1862, el número de suicidios se mantenía constante y era significativamente más bajo que en el resto de Europa, en torno a los 223 casos por año, 1 suicidio por cada 100000 habitantes frente al 12.5 de Francia o al 28.8 de Dinamarca ${ }^{76}$. Sin embargo, constataba que «el número de suicidas había crecido de forma verdaderamente aterradora» en relación a la década anterior, señalando entre sus causas más frecuentes la miseria económica ${ }^{77}$.

\section{EL DEGENERACIONISMO Y SU POSTURA RESPECTO AL SUICIDIO}

El degeneracionismo fue la teoría psiquiátrica que dominó la esfera europea durante las últimas décadas del siglo XIX. Planteaba un modelo dimensional de la enfermedad mental, según el cual los distintos trastornos psíquicos se producen por un proceso común degenerativo ${ }^{78}$. Su fundador, BenedictAuguste Morel, apelaba a una herencia alterada con un sustrato ideológico moral, basado en la ideología católica y simbolizado por el mito del «ángel caído». Su discípulo, Valentín Magnan, defendió un modelo científico natural según el cual la degeneración hereditaria se debía a distintas causas objetivas,

75 VAlentí y ViVó, I (1874), Curso elemental de Medicina Legal. Barcelona, Imp. Verdaguer y compañía, p. 181.

76 Jimeno Agius, J. (1888), El suicidio en España. Madrid, Imp. Góngora y Álvarez, P. 4-5.

77 JiMENo AgIUs (1888), p. 23.

78 Sobre las bases teóricas del degeneracionismo y su influencia en la psiquiatría española tenemos: Plumed, J; REY, A (2002) La introducción de las ideas degeneracionistas en la España del siglo XIX. Aspectos conceptuales. Frenia, II (I), pp. 31-48. 
entre las que destacaban distintas noxas relacionadas con enfermedades de origen social (entre las que el alcoholismo fue un paradigma) ${ }^{79}$. A la hora de conceptualizar el suicidio y el concepto ya caduco de monomanía suicida, Valentín Magnan estableció un cambio a un modelo nosológico en el que la supuesta enfermedad mental no sería sino un síntoma dentro de otros estados patológicos ${ }^{80}$. Este modelo fue asimilado por los psiquiatras españoles en su aproximación al suicidio, que fue conceptualizado sistemáticamente como un síntoma psiquiátrico.

La etiología del cuadro, de acuerdo a los principios de la degeneración, se consideraba debida a la herencia ${ }^{81}$ y la terapia consistía en la toma de medidas higiénicas de intervención social e individual. Así, Jesús Sarabia pensaba que, dado que la herencia era la causa más importante del suicidio, la forma de erradicarlo a nivel social era establecer mejoras en la situación económica de la población y promocionar la enseñanza y la moralidad ${ }^{82}$. M. Zaragoza consideraba el verdadero suicidio un rasgo hereditario. Para prevenirlo, propugnaba evitar ingresar en familias con antecedentes. Sin embargo, si la unión era un hecho irreversible, proponía cambiar la constitución del individuo con medios como la educación y el tipo de alimentación. Para este autor, las medidas individuales resultaban insuficientes y proponía que el Gobierno castigase obras inmorales y el juego, problemas sociales relacionados con el suicidio. Además, sugería medidas preventivas de intervención eliminando aquellos medios habitualmente utilizados por los suicidas, como el fósforo de las cerillas ${ }^{83}$.

El suicidio, pues, fue consagrado como enfermedad social por el degeneracionismo y su prevención se añadió al ideario del movimiento regeneracionista posterior al Desastre del 98. En este sentido, Ambrosio Tapia alertaba en su libro sobre el peligroso aumento del suicidio, definido como mal social, en España en 1897, que relacionaba con vicios sociales como la vagancia, el alcoholismo y el juego. Planteaba a los gobernantes la aplicación de remedios

79 Sobre este tema ver: HuERTAS, R (1992), Madness and degeneration. From «Fallen angel» to mentally ill. I. History of Psychiatry, 3, pp. 391-411.

80 Magnan, V. (1887), Consideraciones generales sobre la locura. Siglo Médico, 34, 104-106; 118-20.

81 Martínez VAlVerde, J (1900), Guía del diagnóstico de las enfermedades mentales, Barcelona, José Espasa, pp. 211-12.

82 SARABIA, J. (1889), El suicidio como enfermedad social. Madrid, Imp. De la Sociedad Montera, p. 21.

83 ZaRAgOZa AveÑo, M. (1877), El suicidio en relación con las ciencias médicas. Discurso manuscrito leído en la Facultad de Medicina de la Universidad Central de Madrid, no publicado, pp. 28-37. 
«a fin de lograr, si no su extirpación completa,»»»al menos que se aminore, ya que su aumento significaría un paso atrás trascendentalísimo en el progreso moral de esta desgraciada España, cuya prosperidad han de conocer seguramente los hombres del siglo XX»; «Parece que la era de la regeneración es llegada $\rangle^{84}$.

\section{CONCLUSIONES}

El proceso de medicalización del suicidio en España se desarrolló a lo largo del siglo XIX. Dado el gran peso de la Iglesia Católica en España a nivel social y político, el proceso fue más conflictivo que en otros países como Francia e Inglaterra. Sin embargo, los frenópatas mostraron su interés desde el comienzo en definir médicamente esta conducta. El cambio se consolidó en la segunda parte del siglo, en el que hubo un acuerdo desde la psiquiatría en considerarlo una enfermedad con un sustrato orgánico. En las dos últimas décadas el degeneracionismo incorporó al suicidio en el grupo de conductas impulsivas patológicas y fue entendido como una manifestación más del proceso degenerativo.

Si desde el principio se relacionó al suicidio con las causas sociales, de acuerdo con el esquema tomado de la psiquiatría francesa, su carácter de enfermedad social fue cobrando mayor peso progresivamente hasta que se consolidó. Aunque inicialmente el factor social más importante relacionado con el suicidio fue el laicismo, finalmente se añadieron otros muchos para los que se propusieron distintas medidas de intervención pública.

Recibido: 14 de junio de 2010.

Aceptado: 2 de marzo de 2011.

84 TAPIA, A. (1900), Los suicidios en Cataluña y, en general, en toda España. Barcelona, Luis Tasso, p. 234. 